\title{
EchoGéo
}

$24 \mid 2013$

Politiques et pratiques de la résilience

\section{Utiliser la polysémie de la résilience pour comprendre les différentes approches du risque et leur possible articulation}

Magali Reghezza-Zitt

\section{(2) OpenEdition}

Journals

Édition électronique

URL : https://journals.openedition.org/echogeo/13401

DOI : 10.4000/echogeo.13401

ISSN : 1963-1197

Éditeur

Pôle de recherche pour l'organisation et la diffusion de l'information géographique (CNRS UMR 8586)

Référence électronique

Magali Reghezza-Zitt, « Utiliser la polysémie de la résilience pour comprendre les différentes approches du risque et leur possible articulation », EchoGéo [En ligne], 24 | 2013, mis en ligne le 10 juillet 2013, consulté le 11 août 2021. URL : http://journals.openedition.org/echogeo/13401 ; DOI : https://doi.org/10.4000/echogeo.13401

Ce document a été généré automatiquement le 11 août 2021.

EchoGéo est mis à disposition selon les termes de la licence Creative Commons Attribution - Pas d'Utilisation Commerciale - Pas de Modification 4.0 International (CC BY-NC-ND) 


\title{
Utiliser la polysémie de la résilience pour comprendre les différentes approches du risque et leur possible articulation
}

\author{
Magali Reghezza-Zitt
}

Le succès de la résilience comme outil opérationnel et concept scientifique se traduit par une sensible augmentation des publications dans le domaine des cyndiniques ${ }^{1}$ (Comfort et al., 2010), sciences qui traitent du risque et des catastrophes (Kervern et Rubise, 1991; Kervern, 1995) et, en particulier, dans la géographie des risques (Dauphiné et Provitolo, 2007 ; Pierdet et Fort, 2010 ; Reghezza et al., 2012). Dans ces travaux, la résilience est tantôt traitée comme un objet d'étude, avec des recherches théoriques visant à éclairer sa (ou plutôt ses) définition(s), ses usages, ses apports heuristiques, méthodologiques et pratiques (Djament et Reghezza, 2012; etc.), ses limites scientifiques mais aussi éthiques et politiques, tantôt comme un volet supplémentaire de l'analyse du risque, qui vient compléter les approches existantes autour de l'aléa, des enjeux, de l'endommagement, de la vulnérabilité, etc. (Serre, 2011 ; Toubin et al., 2012 ; etc.) dans une perspective souvent très appliquée. Dans ce cas, la résilience fait généralement l'objet d'une définition moins rigoureuse et le sens du terme est rarement discuté : la résilience est en effet considérée comme une solution et non comme un problème, solution d'autant plus pertinente que, du fait de la plasticité du mot, elle peut être utilisée dans la continuité, le prolongement des paradigmes existants.

2 Notre propos n'est pas ici de proposer une énième définition de la résilience qui ne ferait vraisemblablement qu'ajouter à la confusion et à la controverse, ni de livrer une critique en règle d'un concept sur lequel il y aurait pourtant beaucoup à dire (Reghezza et al., 2012; Rufat, 2012; Hernandez, 2010 et 2012). La polysémie, le flou terminologique, le manque de rigueur dans l'utilisation (Klein et al., 2004 ; Manyena, 2006 ; Brand et Jax, 2007) sont en effet à l'origine de multiples faiblesses et 
contradictions qui autorisent, une fois le concept sorti du champ académique, des formes de subversion et d'instrumentalisation produisant des effets pervers d'autant plus difficiles à percevoir que la résilience est de prime abord positivement connotée (Rufat, 2012, p. 196-197 ; Provitolo, 2012).

Nous souhaitons en revanche nous appuyer sur cette pluralité des sens, pour montrer deux choses. En premier lieu, cette polysémie est autant la conséquence de l'appropriation rapide de la notion ${ }^{2}$ par des acteurs d'origine et de culture très différentes (Cutter et al., 2008; De Bruijne et al., 2010), que le révélateur d'une ambiguïté fondamentale dans les conceptions qui guident aujourd'hui la compréhension et la gestion des risques. Or, et c'est notre second point, cette polysémie n'invalide pas la pertinence du concept : il est même possible d'articuler les différents sens de la résilience à condition d'expliciter à chaque fois à quel paradigme se rattache telle ou telle acception.

\section{Deux paradigmes distincts pour penser le risque, deux conceptions de la résilience}

On peut, de façon certes schématique, distinguer deux façons de concevoir les risques dans les cindyniques actuelles. Celles-ci correspondent à deux traditions qui se sont construites en parallèle, se sont croisées, ont emprunté l'une à l'autre sans toutefois se rejoindre complètement. Il existe une abondante littérature sur l'épistémologie des cindyniques (Veyret, 2003, p. 6-10 ; Morel et al., 2006 ; etc.) que nous nous proposons ici de relire, non pas selon la chronologie, qui met en évidence la lente et complexe construction des paradigmes, mais selon le résultat de cette construction.

\section{Le risque comme endommagement potentiel : la résilience dans les risk research}

5 La première conception reste dominante, même si elle a été beaucoup critiquée dès les années 1970 (Wisner et al., 1976 ; Dauphiné et Provitolo, 2007). Ce paradigme a pu être qualifié de risk research dans le monde anglophone et est rattaché en France à une culture du risque dite "de l'ingénieur » - par un raccourci très réducteur, qui reflète néanmoins la part de la technique et le rôle de ces acteurs dans sa construction.

\section{Penser le risque à travers la question des dommages}

6 Cette approche a pour caractéristique d'envisager le risque à travers la question des dommages. Elle repose en effet sur l'idée que l'aléa porte atteinte à l'intégrité physique des personnes et des biens. On a d'ailleurs pu parler à un moment d'approche par les impacts (impact approach).

7 Dans ce paradigme, connaître le risque, c'est identifier et décrire les conséquences de l'aléa, donc les dommages, et mesurer la valeur de ces derniers (c'est le principe de l'actuariat). En réponse, le progrès des sciences doit permettre de contenir, voire d'éradiquer, cet endommagement, selon l'idéal de maîtrise et de contrôle d'une nature radicalement extérieure à la société. Dans ce cadre, le dommage est bien au centre des préoccupations, l'action sur l'aléa étant vue comme un instrument au service de la préservation des personnes et des biens. 
8 La source de danger est alors considérée comme un élément indésirable qu'il convient d'expulser du territoire, ou à défaut de contenir dans une logique toute militaire (on parle de front des inondations, de lignes de défense), pour protéger les personnes et les biens (devenus des « enjeux » dans le vocabulaire cindyniste). Dans le cas où il serait impossible d'empêcher l'aléa de sévir, l'augmentation de la résistance physique des enjeux exposés et l'érection de dispositifs de protection s'imposent.

9 Ces trois volets (action sur l'aléa, augmentation de la résistance physique et réduction de l'exposition par les infrastructures de protection) se complètent et trouvent leur aboutissement dans des protocoles de gestion qui relèvent tous de la même logique techniciste et technocratique (prise de décision top down, appuyée sur la rationalité économique, mise en œuvre de l'ingénierie technique (infrastructures de protection, normes architecturales, matériaux adaptés) et juridique (réglementation et zonage), etc.). On voit par ailleurs se dessiner en contre-point les notions de résistance et de reconstruction (qui vient pallier l'échec de la technique à empêcher la destruction), qui sont au fondement de certaines définitions de la résilience.

\section{Une approche focalisée sur le matériel}

10 Cette approche est profondément matérialiste. On s'intéresse avant tout à l'endommagement physique, matériel, qui est vu comme l'origine des autres formes de dommages et, au bout du compte, de l'effondrement sociétal qui caractérise la catastrophe. On pense alors le risque à partir des bâtiments, des infrastructures, des composantes, éventuellement des corps humains (ce qui explique aussi la non-prise en compte des dommages psychologiques dans les évaluations).

11 Il faut souligner ici que la focalisation sur le "matériel » est datée et située. Elle correspond à une culture du risque récente, élaborée dans le cadre de la modernité occidentale et diffusée ensuite à l'échelle internationale. Une comparaison entre les modes de gestion actuels en France et ceux des sociétés rurales des Pyrénées au XVIII ${ }^{\mathrm{e}}$ siècle (Meschinet de Richemond et Reghezza, 2010) a par exemple montré à quel point la place du matériel pouvait varier selon les sociétés et les époques. Cela ne signifie pas que les sociétés pré-modernes aient été indifférentes aux destructions physiques : elles leur donnent cependant une autre signification, une valeur différente, elles n'en font pas une priorité. Contrairement à l'idée répandue, il existe une gestion du risque active dans ces sociétés, mais le but de cette gestion n'est pas de protéger le matériel pour luimême : c'est de permettre la survie et la pérennité de la société (dans le cas étudié, en assurant la continuité de la fonction agricole vivrière du territoire). La préservation du matériel n'est donc pas poursuivie comme fin en soi mais est entièrement subordonnée au projet plus large de reproduction des systèmes sociaux au sein d'un territoire donné.

\section{Des implications méthodologiques importantes qui appellent une certaine définition de la résilience}

12 Penser le risque à partir de l'endommagement implique un certain nombre de positions théoriques et méthodologiques. Le risque est défini comme un endommagement potentiel, donc à partir de l'espérance de dommage (risque = probabilité d'occurrence $\mathrm{x}$ valeur de l'enjeu). Cette approche présuppose une démarche analytique : on décompose l'endommagement potentiel en évaluant les dommages pour chaque enjeu menacé. Ces derniers sont généralement identifiés à partir de leur exposition directe, ce qui est 
exprimé dans la définition risque = aléa + vulnérabilité, et par la pratique qui consiste à superposer la carte d'aléa à celle de l'occupation des sols (Pigeon, 2005).

Depuis plusieurs années, ces méthodes ont été infléchies pour prendre en compte différentes critiques. La complexification croissante des risques, avec en particulier la mise en évidence d'effets dominos, qui décalent les effets des perturbations dans le temps et l'espace et favorisent les mécanismes de diffusion rapide, a montré les limites d'une approche strictement analytique.

La réponse apportée a été double.

1. D'une part, la notion de dommages a été précisée : on distingue aujourd'hui les dommages directs des dommages indirects, ce qui permet de prendre en compte les endommagements par « contagion », pour des enjeux qui se situent hors des espaces initialement impactés. On distingue également les dommages matériels des dommages fonctionnels, ce qui permet d'introduire la notion de continuité d'activité - qui est une autre façon de définir la résilience -, notion essentielle dans les cas d'activité de services métropolitains par exemple, pour lesquels les coûts de l'endommagement du matériel sont infiniment moindres que ceux d'une interruption de l'activité.

Cette distinction est particulièrement développée dans le cas des infrastructures dites critiques (O'Rourke, 2007), qui assurent le fonctionnement quotidien des territoires (fourniture d'eau, d'énergie, télécommunications et transports en particulier) et conditionnent la gestion d'urgence (Gleyze, 2005 ; Lhomme, 2012). Toutefois en pratique, le dommage fonctionnel reste pensé à partir du dommage matériel et de l'exposition directe au danger. La résilience comme continuité d'activité est ainsi subordonnée aux propriétés de résistance et au caractère redondant de certaines infrastructures matérielles qui leur confère une organisation plus adaptable.

2. La seconde réponse consiste en la promotion d'une approche du risque dite "systémique ». Il apparaît en effet que dans certains cas, la somme des dommages ponctuels n'équivaut pas à l'endommagement global. Du fait des effets dominos et des perturbations fonctionnelles, il existe des rétroactions positives et négatives qui augmentent ou diminuent le coût total des sinistres. Plus le territoire est étendu, plus il est hétérogène, plus l'ensemble des enjeux étudiés est complexe, moins la somme des éléments qui les composent est assimilable au tout. L'approche systémique vise alors à adopter une lecture transversale, synthétique et holistique qui intègre les différents types de dommages, les temporalités de ces endommagements, les effets dominos. Dans ce cadre, la résilience apparaît comme un concept particulièrement approprié puisqu'elle s'applique en écologie à des systèmes complexes auto-organisés. Son utilisation renvoie ici à la question de l'équilibre du système. La résilience comme " retour à la normale ", qui est une autre façon de définir le terme, peut en effet être envisagée comme un retour à l'équilibre antérieur à la perturbation (ce qui suppose la recherche d'un système en équilibre stable avec un état de référence et une approche fixiste) ou comme le passage à un nouvel état d'équilibre, avec en particulier l'intégration des leçons de la crise (retour d'expérience) et l'adaptation du système (ce qui demande d'interroger les seuils à partir duquel l'adaptation conduit à une bifurcation, mais implique aussi de considérer les systèmes étudiés comme en équilibre fondamentalement instable).

\section{Du dommage potentiel au potentiel d'endommagement : la résilience dans les hazard research} tout à la fois la précédente. Elle a été développée dès le début du $\mathrm{XX}^{\mathrm{e}}$ siècle à partir 
d'une réflexion sur le rapport environnement/société. Afin de dépasser le déterminisme mésologique qui prévalait dans l'explication des relations homme/ nature, les géographes américains de l'université de Chicago ont développé plusieurs concepts : resources, hazard, vulnerability (Chorley, 1973; Cutter, 1994 ; Reghezza, 2006) qui ont servi de fondement à l'élaboration de paradigme. Dans les sciences sociales françaises, les travaux de Georges-Yves Kervern (Kervern et Rubise, 1991), lorsqu'il dissocie risque et danger, ceux de Jean-Louis Fabiani et Jacques Theys (Fabiani et Theys, 1987) lorsqu'ils introduisent la notion de "société vulnérable ", la thèse pionnière de Robert D'Ercole, les textes de Valérie November (November, 2002) ou de Patrick Pigeon (Pigeon, 2005) (la liste n'est évidemment pas exhaustive!) contribuent à diffuser cet autre regard sur le risque.

\section{Penser le risque à travers l'interaction environnement société}

Le fil directeur de ces travaux n'est pas le risque mais la compréhension des interactions réciproques entre la société et son environnement (White et al., 1978). S'inspirant de l'écologie botanique développée aux États-Unis à la fin du XIXe siècle, ces chercheurs se réapproprient le concept d'adaptation pour l'opposer à celui d'influence, car ce dernier implique une causalité linéaire qui ne peut rendre compte de la complexité des relations entre les sociétés et leur environnement. Le concept de hazard permet tout à la fois d'illustrer et formaliser la complexité de ces relations.

17 Les réflexions sur l'adaptation débouchent sur la notion de coping capacity, capacité à faire face qui explique la réaction différenciée des sociétés, groupes et individus aux menaces et aux perturbations. Chaque société, chaque groupe, chaque individu oppose en effet un type de réponse(s) à un aléa et à son occurrence.

18 Ces réponses sont fonction de divers paramètres (sociaux, économiques, politiques, techniques, individuels ou collectifs, etc.) qui varient dans le temps et l'espace, et qui définissent sa capacité à faire face ou non. On arrive alors à la vulnérabilité, concept qui subsume les idées d'adaptation, de capacité à faire face voire de résilience (White et al., 1978 ; Blaikie et al., 1994 ; Cutter et al., 2003).

Le déplacement de la focale sur la vulnérabilité demande d'ailleurs de réenvisager le rapport entre crise et catastrophe: si la perturbation entraîne une situation de désorganisation, une rupture dans le quotidien, qui peut être qualifiée de crise, celle-ci ne se transforme pas nécessairement en catastrophe si la réponse de la société est appropriée. Au-delà de la question des seuils, objectifs et subjectifs, qui permettent de dire si tel ou tel événement relève de la catastrophe, l'entrée par la vulnérabilité permet de déconstruire une vision linéaire de la catastrophe, qui la rattacherait mécaniquement à l'occurrence de l'aléa et à l'endommagement matériel que ce dernier produit.

\section{L'entrée par la vulnérabilité : un changement de paradigme}

20 Cette entrée par la vulnérabilité exprime en fin de compte, un décentrement du regard porté sur les catastrophes: alors que les risk research se concentrent sur le risque comme endommagement potentiel, les hazard research mettent en exergue la vulnérabilité comme potentiel d'endommagement. Ce n'est plus le dommage qui est au centre, mais l'explication des dommages à partir de facteurs d'endommagement qui sont à la fois endogènes et exogènes (D'Ercole et al., 1994 ; Reghezza, 2006). 
21 Cette distinction est explicitement formulée par R. D’Ercole et J.-C. Thouret, lorsqu'ils écrivent en 1996 qu'il existe deux approches de la vulnérabilité : « la classique consiste à mesurer l'endommagement potentiel des éléments exposés ; la nouvelle, intégrée et complémentaires de la première vise à cerner les conditions ou les facteurs propices aux endommagements ou influant sur la capacité de réponse à une situation de crise » (D’Ercole et Thouret, 1996, p. 407).

1. Dans le premier cas, on parle certes de vulnérabilité, mais comme synonyme de fragilité, de sensibilité, ce qui traduit à nouveau le rapport au physique et au matériel. On retrouve bien l'idée de mesure, qui se traduit dans la fabrication de nombreux indicateurs, et de dommage avec un glissement du risque à la vulnérabilité, si bien que parfois on finit par se demander si les deux termes ne sont pas interchangeables. La vulnérabilité est un état, sur lequel on peut agir par des solutions essentiellement techniques.

2. Dans le second, la vulnérabilité est une propriété, un processus : l'enjeu est actif, ce qui ouvre la voie à l'idée d'adaptation proactive exprimée dans certaines définitions de la résilience (Comfort et al., 2010). Dans le premier paradigme, on adapte l'enjeu pour qu'il résiste. Dans le second, l'enjeu s'adapte par réaction. Par ailleurs, dans ce second paradigme, on s'interroge finalement moins sur le risque que sur la catastrophe (risque réalisé) et sur le système complexe de causalités qui l'expliquent et finalement la produisent. On est bien dans la recherche des facteurs, endogènes et exogènes, collectifs et individuels, structurels et contingents, etc. Ainsi, dans les années 1990, les modèles développés par Piers Blaikie et al. rappellent que le risque est d'abord une situation qui met en jeu de nombreux niveaux de causalité, qui vont expliquer a posteriori pourquoi la catastrophe a eu lieu (Blaikie et al., 1994). L'identification de ces causes permet ensuite d'identifier des situations de risque analogue et d'anticiper, a priori, la survenance des catastrophes.

\section{Une autre façon de penser la résilience}

Cette distinction est manifeste en anglais puisqu'on utilise deux termes différents :hazard, que l'on traduit à tort par aléa, n'est pas synonyme de risk. Risk désigne en effet une mesure quantitative d'une incertitude (en l'occurrence, du dommage potentiel), tandis que hazard qualifie le produit de l'interaction entre une société et son environnement.

Cette interaction peut produire aussi bien des ressources que des dommages (le hazard étant explicitement défini comme le pendant négatif de la ressource (White et al., 1978)): selon les moments, un hazard peut d'ailleurs constituer une ressource et réciproquement. Si cette idée n'est pas absente du paradigme précédent, les espérances de dommages pouvant aussi intégrer les bénéfices au calcul des coûts, la focalisation sur le dommage impose de mettre de côté l'aspect positif du risque et de la catastrophe : dans cette perspective, la vulnérabilité ne peut être vue que comme une propriété indésirable, négative, la résilience étant alors pensée comme le pendant positif (flipside) et recherché de cette dernière (Kasperson et Kasperson 2001 ; Lhomme et al., 2010 ; Reghezza et al., 2012).

En revanche, dans le second paradigme, puisque la vulnérabilité est un construit, un processus (au sens où cette propriété intrinsèque se modifie constamment en fonction de facteurs endogènes et exogènes), vulnérabilité et résilience peuvent s'inscrire dans un rapport plus complexe (Gallopin, 2006), certains facteurs de vulnérabilité pouvant devenir des facteurs de résilience. C'est ce que formalise par exemple Damienne Provitolo dans le terme de « vulnérabilité résiliençiaire » (Provitolo, 2012). 
Illustration 1 - La résilience, deux paradigmes distincts mais complémentaires

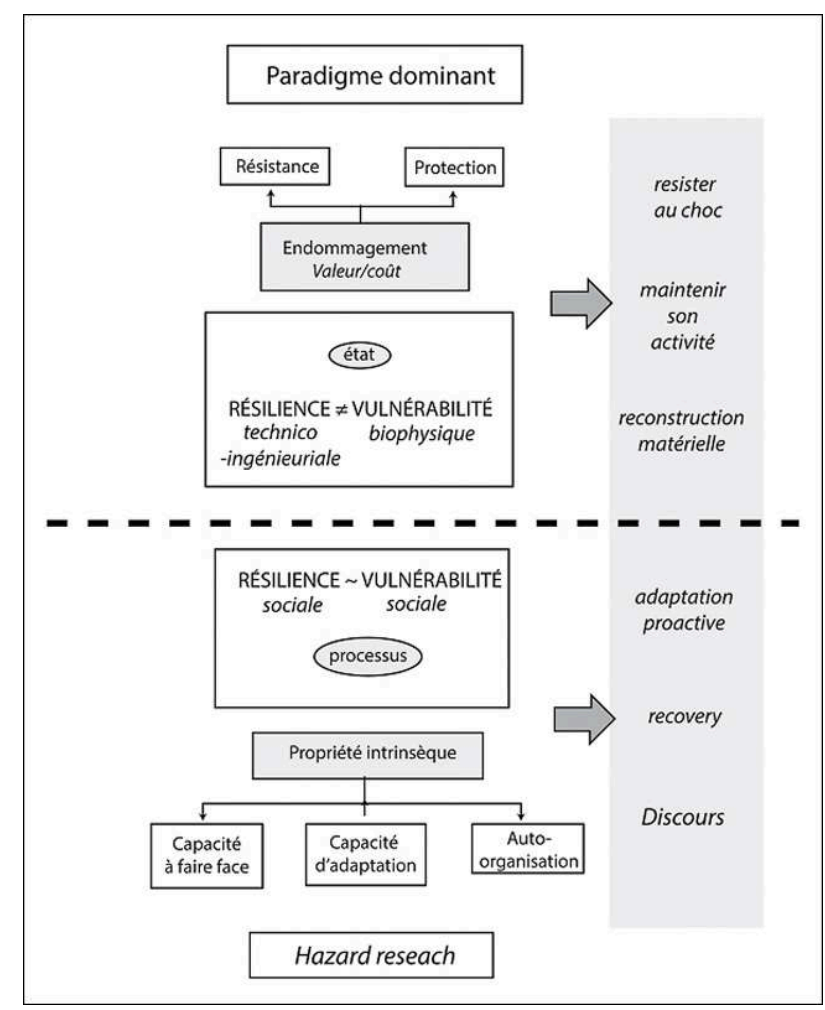

Source : M. Reghezza, d'après M. Reghezza et D. Provitolo, travaux en cours.

\section{Repenser la résilience en explicitant les paradigmes}

En distinguant les deux paradigmes (cf. illustration 1), nous avons pu retrouver, au fur et à mesure, la multiplicité d'acceptions du terme « résilience » (Serge Lhomme utilise par exemple le terme de "nébuleuse » (Lhomme et al., 2010)), qui s'inscrivent chacune dans un contexte et un usage précis. Cette distinction peut sembler factice dans la mesure où chaque paradigme ne cesse d'emprunter à l'autre de façon plus ou moins consciente. Elle n'existe d'ailleurs plus en tant que telle dans les pratiques.

\section{Lever les contradictions}

Si l'on admet que la résilience recouvre en réalité sous un seul vocable deux paradigmes différents, alors on peut lever la contradiction entre certaines acceptions. Serge Lhomme pointe par exemple - à juste titre - l'incompatibilité entre d'un côté, la résilience comme résistance et de l'autre, la résilience comme flexibilité et souplesse qui permettent l'adaptabilité. Cette contradiction est levée si l'on pose que chaque usage du terme répond à un besoin précis, cohérent avec le paradigme dans lequel il s'inscrit.

Si en effet la gestion obéit à la logique du "zéro dommage " (ou son avatar, du dommage minimal) et considère l'aléa comme un élément extérieur au système sociotechnique, alors l'intégrité physique des enjeux est une priorité et la focalisation sur le matériel une nécessité logique. 

l'aléa qui déstabilise le système. À défaut de pouvoir empêcher le système de quitter son état d'équilibre, l'objectif est de le ramener à un nouvel état d'équilibre, identique ou du moins le plus proche possible de l'état initial. La crise ne peut être qu'une parenthèse qui doit être refermée au plus vite, car elle sanctionne l'échec des mesures mises en œuvre qui visent justement à l'empêcher. Dans ce cadre, la propriété de résistance est une façon de s'opposer à l'aléa et donc de limiter les dommages et la crise/catastrophe qui en résulte. Elle peut ensuite se décliner dans son volet biophysique (affaiblissement de l'aléa, augmentation de la résistance du bâti, réduction de l'exposition) ou social (augmentation de la résistance par amélioration du potentiel organisationnel des secours et de l'urgence).

logique n'est plus la même: la crise n'est pas une rupture mais un temps fort de la trajectoire territoriale, qui appelle une réponse $\mathrm{du}$ système et explique sa transformation. Dans cette optique, le système est en équilibre instable par nature : les états d'équilibre se succèdent et le système s'adapte en permanence $\mathrm{au}(\mathrm{x})$ changement(s). L'acceptabilité du dommage matériel ne relève pas du fatalisme mais d'une autre hiérarchie des valeurs. On retrouve ici la logique mise en évidence dans le cas des sociétés pré-modernes évoqué plus haut : ce n'est pas tant la reconstruction que l'absorption de la perturbation, pour permettre la reproduction sociale et spatiale du territoire, qui compte.

Par conséquent, alors que dans un cas, la reconstruction est focalisée sur le matériel (ce qui explique de rôle de l'indemnisation par les assurances par exemple), dans l'autre, la résilience signifie avant tout le rétablissement fonctionnel. De fait, la propriété de résistance n'est pas écartée, mais elle n'occupe pas la même position dans le dispositif gestionnaire.

\section{Repenser la résilience en fonction des temporalités du risque et de la crise : réconcilier les paradigmes?}

31 On peut alors repositionner les différents sens de la résilience, en les rattachant chaque fois au paradigme correspondant.

Partons de la situation de risque, qui précède l'occurrence de la perturbation. Celle-ci entrâne un déséquilibre du système: c'est le temps de l'urgence qui suit immédiatement l'occurrence de l'aléa. La désorganisation qu'elle entraîne est fonction du degré d'endommagement et de la capacité de réponse des organisations mises en place pour gérer cette urgence. Si la réponse est adaptée, la perturbation est rapidement absorbée et le système retrouve un état d'équilibre: on a affaire à un incident ou un accident. Dans le cas contraire, si le seuil d'endommagement est trop important et/ou si la réponse sociale est inadaptée, la situation se transforme en crise.

Cette crise a sa propre temporalité qui dépend du temps de retour à la «normale ", c'est-à-dire à une situation dans laquelle le système regagne un état d'équilibre, proche ou éloigné de l'état initial, mais qui lui permet de retrouver une stabilité. La crise a une durée variable, qui dépend autant de la nature de l'aléa que de la capacité de la société à faire face. La crise peut elle-même se décomposer en deux temps, qui se chevauchent : 
le temps de l'urgence, où le système doit faire face au choc et celui où il amorce la sortie de crise et commence sa récupération.

On a alors trois moments distincts qui mobilisent chacun des sens différents de la résilience. Et il est frappant de constater que les deux paradigmes évoqués plus haut ne sont pas convoqués aux mêmes moments.

1. Au temps de l'impact, la résilience peut s'entendre comme la capacité à résister au choc. La propriété de résilience dépend essentiellement de la propriété de résistance matérielle du système. On est bien ici dans le premier paradigme. La résilience s'oppose alors effectivement à la vulnérabilité : plus l'enjeu est vulnérable, plus il est susceptible de subir un dommage. Or, c'est l'ampleur et la nature de ces dommages qui placent le système en situation de déséquilibre et de crise. En ce sens d'ailleurs, la perturbation est moins l'aléa que l'effet que cet aléa produit sur un système biophysiquement vulnérable. Ce sens permet de conforter l'action préventive: en diminuant la vulnérabilité, on augmente mécaniquement la résilience.

2. Immédiatement après la déstabilisation du système, soit dans le temps de l'urgence à proprement parler, la résilience s'entend comme la capacité à se maintenir malgré le choc, à l'absorber et à quitter la situation d'instabilité pour revenir à l'état d'équilibre. La propriété de résilience dépend ici de trois capacités: la capacité de continuité des fonctions du système territorial et de ses sous-systèmes socio-techniques, même en mode dégradé; la capacité de restauration rapide des fonctions stratégiques du système lorsqu'elles sont endommagées ou arrêtées; la capacité à mettre en œuvre des stratégies de retour à la normale.

On note ici que la résilience n'est plus directement et exclusivement liée au dommage matériel, même si le dommage matériel peut avoir des conséquences fonctionnelles. On est ici à cheval sur les deux paradigmes. Comme la vulnérabilité, la résilience dépend de facteurs biophysiques, sociaux (en particulier organisationnels) et spatiaux, qui se mettent en place avant et pendant la crise, sans qu'il y ait d'ailleurs forcément opposition entre les déterminants de la résilience et ceux de la vulnérabilité. Ainsi, la concentration des biens et des personnes est un facteur d'endommagement accru, qui détermine une vulnérabilité biophysique forte, mais elle appelle aussi une mobilisation des moyens de secours plus importante: le récent exemple de New York et du cyclone Sandy a ainsi montré la focalisation des moyens (et des médias) sur le centre métropolitain au détriment d'autres espaces urbains, périurbains et ruraux.

3. Une fois la sortie de crise amorcée, la résilience renvoie au processus de recovery, qu'on pourrait traduire par l'idée de récupération. Nous préférons à dessein le terme anglais car il est beaucoup plus riche que ses traductions françaises et recouvre à lui seul plusieurs sens du mot résilience.

Recovery peut d'abord désigner la reconstruction, c'est-à-dire la remise en état du matériel. On retrouve ici le premier paradigme. La résilience comme reconstruction, c'est-à-dire une restauration matérielle, est autant un état qu'un processus, mais n'est plus une propriété. On peut d'ailleurs mesurer cet état par rapport à un état de référence. La reconstruction dépend de la vulnérabilité biophysique du système sans toutefois être entièrement déterminée par elle. On peut être matériellement très vulnérable et se reconstruire très vite. C'est ce que signifie de façon certes provocatrice Samuel Rufat lorsqu'il indique que la forme la plus résiliente est le bidonville, car, du fait des matériaux utilisés, il est éminemment fragile mais peut se rebâtir très rapidement (Rufat, 2012).

Recovery désigne toutefois un processus beaucoup plus large que la reconstruction matérielle : c'est alors le second paradigme qui est mobilisé. Si l'on examine les travaux de Julie Hernandez sur la Nouvelle-Orléans (Hernandez, 2009), on remarque qu'elle distingue la reconstruction de l'urbs et de la civitas: d'un côté, le fait de rebâtir matériellement la 
Nouvelle-Orléans, de l'autre, de reconstruire le lien social, le faire société. On sort bien de la logique du système socio-technique pour envisager un système social territorialisé.

Dans cette perspective, la résilience n'est plus seulement un donné objectif, mesurable par des indicateurs au même titre que le risque et la vulnérabilité. Elle est aussi un discours qui acte la fin d'un processus et l'état qui en résulte. La question est notamment de savoir qui décide, à quel moment et pourquoi tel ou tel système doit être considéré comme résilient. La résilience n'est pas non plus forcément positive : les notions de reproduction et de pérennité, qui permettent de redonner du champ temporel, d'historiciser la résilience comme le propose Géraldine Djament (Reghezza et Djament, 2012), sont ici utiles, car l'on voit que le processus de recovery peut renouveler des situations indésirables, des fractures sociales et spatiales, des injustices, etc.

\section{Conclusion}

Il apparaît donc que les multiples acceptions de la résilience s'inscrivent en totale cohérence avec les paradigmes auxquels elles se rattachent, dont elles découlent de façon nécessaire : elles ne sont pas contradictoires mais révèlent différentes approches d'un même objet, différentes logiques qui à un moment donné entrent en contradiction. De fait, nous postulons que la résilience n'ouvre pas la voie à un nouveau paradigme mais que sa polysémie sanctionne la coexistence de deux paradigmes, certes complémentaires mais fondamentalement distincts et que, si elle permet un renouvellement des pratiques au sein de ces paradigmes, elle ne s'inscrit pas dans une rupture mais au contraire dans un prolongement. Lever les contradictions oblige en revanche à expliciter chaque position scientifique, chaque conception. En ce sens, de même que la vulnérabilité a pu être utilisée comme une clé de lecture particulièrement efficace des sociétés et des territoires, de même la résilience peut être un outil réflexif puissant, pour relire aujourd'hui les paradigmes qui président à la gestion et la compréhension des risques et des catastrophes.

\section{BIBLIOGRAPHIE}

Blaikie P., Cannon T., Davis I., Wisner B., 1994. At Risk: Natural hazards, people's vulnerability and disasters. London, Routledge, $284 \mathrm{p}$.

Brand F. S., Jax K., 2007. Focusing on the meaning(s) of resilience: resilience as a descriptive concept and a boundary object. Ecology and Society, vol. 12, $\mathrm{n}^{\circ} 1,16 \mathrm{p}$.

Comfort L. K., Boin A., Demchak C., 2010. Designing Resilience. Preparing fo Extrem Events. Pittsburgh, University of Pittsburgh Press.

Chorley R. J. (ed), 1973. Directions in Geography. Methuen and Co Ltd, Londre

Cutter S. L., 1994. Environmental Risks and Hazards. Prentice hall., inc. 
Cutter S. L., Boruff B. J., Shirley W. L., 2003. Social vulnerability to environmental hazards. Social Science Quarterly, 84 (1), p. 242-261.

Cutter S. L., Barnes L., Berry M., Burton C., Evans E., Tate E., Webb J., 2008. A place-based model for understanding community resilience to natural disasters. Global Environmental Change, 18(4), p. 598-606.

Dauphiné A., Provitolo D., 2007. La résilience : un concept pour la gestion des risques. Annales de Géographie, n 654, p. 115-125.

De Bruijne M., Boin A., Van Eeten M., 2010. The rise of résilience. In Comfort L. K., Boin A., Demchak C. (eds). Designing Resilience. Preparing fo Extrem Events. Pittsburgh, University of Pittsburgh Press, p. 13-32.

D’Ercole R., 1991.Vulnérabilité des populations face au risque volcanique. Le cas de la région du volcan Cotopaxi (Équateur). Thèse de doctorat, Grenoble, Université Joseph Fourier, 460 p.

D’Ercole R., Thouret J.-C., Dollfus O., Asté J.-P., 1994. Les vulnérabilités des sociétés et des espaces urbanisés : concepts, typologie, mode d'analyse. Revue de Géographie Alpine, vol. 82, nº 4, p. 87-96.

Djament G., Reghezza M. (dir.), 2012. Résiliences urbaines, les villes face aux catastrophes. Éditions du manuscrit, $364 \mathrm{p}$.

Fabiani J.-L., Theys J., 1987. La Société vulnérable : évaluer et maîtriser les risques. Presse de la rue d'Ulm, Paris, 688 p.

Gallopin G. C., 2006. Linkages between vulnerability, resilience and adaptive capacity. Global Environmental Change, vol. 16, $\mathrm{n}^{\circ} 3$, p. 293-303.

Gleyze J.-F., 2005. La vulnérabilité structurelle des réseaux de transport dans un contexte de risques. Thèse de doctorat en Analyse Théorique et Épistémologique en Géographique, Université Paris VII - Denis Diderot, 848 p.

Hernandez J., 2009. The Long Way Home : une catastrophe qui se prolonge à La Nouvelle-Orléans, trois ans après le passage de l'ouragan Katrina. Espace géographique, T. 38, nº 2, p. 124-138.

Hernandez J., 2010. ReNew Orleans ? Résilience urbaine, mobilisation civique et création d'un “capital de reconstruction” à la Nouvelle Orléans après Katrina. Thèse de doctorat, Université Paris X - Nanterre.

Hernandez J., 2012. Résiliences contradictoires et résilience toxique dans la Nouvelle-Orléans post Katrina. In Djament G., Reghezza M. (dir.), Résiliences urbaines, les villes face aux catastrophes. Éditions du manuscrit, p. 204-235.

Kasperson J. X., Kasperson R. E., 2001. International Workshop on Vulnerability and Global Environmental Change. SEI Risk and Vulnerability Program Report, http://www.sei.se/risk/ workshop.html

Kervern G.-Y., Rubise P., 1991. L'Archipel du danger : introduction aux cindyniques. Économica, Paris, $460 \mathrm{p}$.

Kervern G.-Y., 1995. Éléments fondamentaux des cindyniques. Économica, Paris, 110 p.

Klein R. J. T., Nicholls R. J., Frank T., 2004. Resilience to natural hazards: how useful is this concept? Environmental Hazards, vol. 5, p. 35-45.

Lhomme S., Serre D., Diab Y., Laganier, R., 2010. Les réseaux techniques face aux inondations ou comment définir des indicateurs de performance de ces réseaux pour évaluer la résilience urbaine. Bulletin de l'association des géographes français, p. 487-502. 
Lhomme S., 2012, Les réseaux techniques comme vecteur de propagation des risques en milieu urbain. Une contribution théorique et pratique à l'analyse de la résilience urbaine. Thèse de doctorat Universités Paris Diderot et Paris-Est (EIVP).

Manyena S. B., 2006. The concept of resilience revisited. Disasters, vol. 30, n ${ }^{\circ} 4$, p. 434-450.

Meschinet de Richemond N., Reghezza M., 2010. La gestion du risque en France : contre ou avec le territoire? Annales de géographie, $\mathrm{n}^{\circ}$ 673, p. 248-267.

Meschinet de Richemond N., 2012. Risques, crises et territoires. Réflexions géographiques et historiques sur l'histoire des cindyniques. Mémoire d'HDR, vol. 1.

Morel V., Deboudt P., Hellequin A.-P., Herbert V., Meur-Férec V., 2006. Regard rétrospectif sur l'étude des risques en géographie à partir des publications universitaires (1980-2004).

L'Information Géographique, vol. 70, n 1, p. 6-24.

November V., 2002. Les territoires du Risque. Le risque comme objet de réflexion géographique. Peter Lang, Berne, $332 \mathrm{p}$.

O'Rourke T. D., 2007. Critical infrastructure, interdependencies and résilience. The Bridge - The Journal of the National Academy of Science, 37 (1), p. 22-31

Pierdet C., Fort M. (coord.), 2010. Les grandes métropoles au risque de l'eau : mise en crise et résilience spatiale au Nord et au Sud. Bulletin de l'Association de géographes français-Géographies, $\mathrm{n}^{\circ} 4,138 \mathrm{p}$.

Pigeon P., 2005. Géographie critique des risques. Paris, Économica, 217 p.

Provitolo D., 2012. The Contribution of Science and Technology to meeting the Challenge of Risk and Disaster Reduction in Developing Countries: From Concrete Examples to the Proposal of a Conceptual Model of « Resiliencery Vulnerability. In Bolay J.-C. et al. (eds). Technologies and Innovations for Development. Springer-Verlag.

Reghezza M., 2006. Réflexions sur la vulnérabilité métropolitaine. La métropole parisienne face au risque de crue centennale. Thèse de doctorat, Université Paris X-Nanterre.

Reghezza M., Rufat S., Djament G., Le Blanc A., Lhomme S., 2012. What Resilience Is Not: Uses and Abuses. Cybergeo: European Journal of Geography, mis en ligne le 18 octobre 2012, http:// cybergeo.revues.org/25554; DOI : 10.4000/cybergeo.25554

Rufat S., 2012. Existe-t-il une « mauvaise » résilience ? ». In Djament G., Reghezza M. (dir.), Résiliences urbaines, les villes face aux catastrophes. Éditions du manuscrit, p. 158-203.

Serre D., 2011. La ville résiliente aux inondations, méthodes et outils d'évaluation. Mémoire d'HDR, Université Paris-Est, Marne-la-Vallée, 173 p.

Toubin M., Lhomme S., Diab Y., Serre D., Laganier, R., 2012. La Résilience urbaine : un nouveau concept opérationnel vecteur de durabilité urbaine ? Développement durable et territoires, vol. 3 , $\mathrm{n}^{\circ}$ 1, http://developpementdurable.revues.org/9208

Thouret J.-C., d'Ercole R., 1996. Vulnérabilité aux risques naturels en milieu urbain : effets, facteurs et réponses sociales. Cahiers des Sciences humaines, 32 (2), p. 407-422.

Vale L.V., Campanella T. J. (eds.), 2005. The Resilient City. How modern cities recover from disaster. New York, Oxford University Press, 376 p.

Veyret Y. (dir.), 2003. Les risques. Paris, SEDES, 255 p.

White G. F., Burton I., Kates R., 1978. The Environment as Hazard. New York, Oxford University Press. 
Wisner B., O'Keefe P., Westgate K., 1976. Taking the Naturalness out of Natural Disasters. Nature, Vol. 260, n5 5552, p. 566-567.

\section{NOTES}

1. Nous reprenons ici le néologisme introduit par G.-Y. Kervern lors du colloque fondateur qui s'est tenu à la Sorbonne en 1987 dans un sens très général, pour regrouper, par commodité, tout ce qui se rattache aux sciences du danger sous un même vocable. Nous nous éloignons donc du sens précis utilisé par Kervern.

2. Nous utilisons ici à dessein le terme de "notion ", pour le distinguer de celui de "concept» qui renvoie à une construction scientifique. La résilience semble aujourd'hui utilisée pour rendre compte d'une intuition, à savoir que malgré la récurrence des aléas, calamités et autres désastres qui frappaient les sociétés, il était rare de constater une disparition de ces dernières et en tous cas, qu'il était impossible de relier l'effondrement et la catastrophe selon une causalité linéaire mécanique (Vale et Campanella, 2005). La catastrophe est davantage un révélateur, un catalyseur d'effondrement qu'une cause. La disparition prend place dans une trajectoire territoriale, sociale, qui dépend d'un système de causalités complexe et mobilise des temporalités multiples. De fait, avant de recevoir une formalisation scientifique rigoureuse, largement permise par des emprunts et des transferts disciplinaires [on cite habituellement la physique des matériaux, l'écologie botanique et, dans une moindre mesure, la psychologie (Reghezza et al., 2012)], la résilience vient répondre à un constat empirique paradoxal. Elle est donc, en pratique du moins, très souvent utilisée comme une notion et non comme un concept.

\section{RÉSUMÉS}

La résilience est un terme à la mode, qui souffre cependant de sa polysémie. Cet article propose de montrer deux choses. D'une part, cette polysémie est autant le fait de la construction pluridisciplinaire du concept que de la coexistence de deux paradigmes distincts au sein des sciences qui traitent du risque, les cindyniques. Le même mot de résilience relève ainsi deux conceptions du risque différentes, qui si elles ne sont pas exclusives l'une de l'autre, ne sont pas réductibles l'une à l'autre. D'autre part, il est possible de repositionner les différentes acceptions de la résilience en fonction des deux paradigmes pour lever les contradictions. La résilience devient alors un outil réflexif pour penser les cindyniques dans leur diversité et leurs complémentarités.

Resilience is a fashionable buzzword. But the polysemy of the concept may weaken his relevance. This paper aims at demonstrating two points. First, this polysemy is obviously linked with a multidisciplinary construction. However we assume that the polysemy can be explained in another way: one single world expresses two different paradigms. Those paradigms may have some things in common, but they cannot be merged. Secondly, we will show that it is possible to solve the contradictions created by that polysemy, if we reconsider the different meanings of resilience in relation with each paradigm. Resilience is thus a reflexive tool, which allows a better understanding of the risk and hazard research fields. 
INDEX

Keywords : resilience, vulnerability, adaptation, risk, crisis

Mots-clés : résilience, vulnérabilité, adaptation, risque, crise

Thèmes : Sur le Champ - Sur le Terrain

\section{AUTEUR}

\section{MAGALI REGHEZZA-ZITT}

Magali Reghezza-Zitt, reghezza@mercator.ens.fr, est Maître de conférences à l'École Normale Supérieure. Elle a publié récemment :

- Reghezza M., 2012. Résiliences urbaines, les villes face aux catastrophes, Éditions du manuscrit, ouvrage collectif dirigé en collaboration avec G. Djament-Tran.

- Reghezza M., Rufat S., Djament-Tran G., Le Blanc A., Lhomme S., 2012. What Resilience Is Not : Uses and Abuses. Cybergeo, Environnement, Nature, Paysage, document 621, http:// cybergeo.revues.org/25554

- Reghezza M., 2010. La Résilience dans les politiques françaises de gestion des inondations urbaines : quelques pistes d'analyse. Bulletin de l'association de géographes français, $\mathrm{n}^{\circ} 4$, p. 507-516, numéro spécial coordonné par C. Pierdet et M. Fort. 